\title{
Self-calibration: an efficient method to control systematic effects in bolometric interferometry
}

\author{
M.-A. Bigot-Sazy ${ }^{1}$, R. Charlassier ${ }^{2}$, J.-Ch. Hamilton ${ }^{1}$, J. Kaplan ${ }^{1}$, and G. Zahariade ${ }^{1}$ \\ 1 APC, Astroparticule et Cosmologie, Université Paris Diderot, Bâtiment Condorcet, 10 rue Alice Domon et Léonie Duquet, \\ 75205 Paris Cedex 13, France \\ e-mail: mabigot@apc.univ-paris7.fr \\ 2 Turing-Solutions, 25 rue Dauphine, 75006 Paris, France
}

Received 21 September 2012 / Accepted 4 December 2012

\section{ABSTRACT}

\begin{abstract}
Context. The QUBIC collaboration is building a bolometric interferometer dedicated to the detection of B-mode polarization fluctuations in the cosmic microwave background.

Aims. We introduce a self-calibration procedure related to those used in radio-interferometry to control a wide range of instrumental systematic errors in polarization-sensitive instruments.

Methods. This procedure takes advantage of the need for measurements on redundant baselines to match each other exactly in the absence of systematic effects. For a given systematic error model, measuring each baseline independently therefore allows writing a system of nonlinear equations whose unknowns are the systematic error model parameters (gains and couplings of Jones matrices, for instance).

Results. We give the mathematical basis of the self-calibration. We implement this method numerically in the context of bolometric interferometry. We show that, for large enough arrays of horns, the nonlinear system can be solved numerically using a standard nonlinear least-squares fitting and that the accuracy achievable on systematic effects is only limited by the time spent on the calibration mode for each baseline apart from the validity of the systematic error model.
\end{abstract}

Key words. instrumentation: polarimeters - instrumentation: interferometers - cosmic background radiation - inflation methods: data analysis

\section{Introduction}

The quest for the B-mode of the polarization of the cosmic background is one of the scientific priorities of observational cosmology today. Observing this mode appears to be the most powerful way to constrain inflation models. However, detecting such a weak signal is a real experimental challenge. In addition to a high statistical sensitivity (a huge number of horns and bolometers required), future experiments will need excellent quality of foreground removal and unprecedented control of instrumental effects.

Currently, most projects are based on the experimental concept of an imager. A promising alternative technology is bolometric interferometry. This is the project of the QUBIC instrument (QUBIC collaboration 2010). A first module is planned for installation at the Franco-Italian Concordia Station in Dome C, Antarctica in 2014. The aim is to combine the advantages of an imager in terms of sensitivity with those of an interferometer in terms of controlling systematic effects. The statistical sensitivity of the QUBIC instrument is comparable to that of an imager with the same number of horns covering the same sky fraction. The full QUBIC instrument (six modules) will comprise three frequencies $(97,150$, and $220 \mathrm{GHz}$ ) and aims to constrain, at the $90 \%$ confidence level, a tensor-to-scalar ratio of 0.01 with one year of data.

The aim of this article is to introduce a new method, specific to bolometric interferometry, called self-calibration, and to give an example of application with the QUBIC instrument. This method allows a wide range of instrumental systematic effects to be controlled.
This self-calibration technique is based on the redundancy of the receiver array (Wieringa 1991). It uses the need for redundant baselines of the interferometer to measure exactly the same quantity in the absence of systematic effects. For a real instrument, these measurements will be different because of systematics. The small differences can be used to calibrate parameters that characterize the instrument completely for each channel and estimate the instrumental errors.

In the case of a bolometric interferometer, the square horn array will provide a large number of redundant baselines. In this way, a bolometric interferometer can be self-calibrated thanks to a calibration mode during which it will separately measure the $n_{\mathrm{h}}\left(n_{\mathrm{h}}-1\right) / 2$ baselines or a fraction of the $n_{\mathrm{h}}\left(n_{\mathrm{h}}-1\right) / 2$ baselines with $n_{\mathrm{h}}$ the number of horns observing an external polarized source.

This method is inspired by traditional interferometry (Pearson \& Readhead 1984) where signal phases are often lost due to atmospheric turbulence. Standard calibration procedures exist in radio-interferometry and are similar to those used for imaging techniques based on observations of an unresolved source whose flux is assumed to be known. We emphasize that the word self-calibration refers, by opposition, to a procedure in which no knowledge of the observed source is required (most of the time, the object which is scientifically studied is itself used as the calibration source). Most of these self-calibration techniques are based on the evaluation of so-called closure quantities phases or amplitudes. A set of unknown phases can, for instance, be iteratively reconstructed by forming quantities where they are nullified (the product of the three visibilities that can 
be formed with three antennas). The use of redundant baselines for calibration is in contrast rather uncommon in radiointerferometry (see Wieringa 1991; Noordam \& de Bruyn 1982). This comes from most radio-interferometers having very few redundant baselines achieve very high angular resolution, it is indeed better to arrange a given number of antennas in order to optimize the uv-plane sampling, rather than to maximize redundancy.

A new kind of all-digital radio-interferometer the "omniscope", dedicated to $21 \mathrm{~cm}$ observations, has recently been proposed by Tegmark \& Zaldarriaga (2009, 2012); its concept can be summarized by the following five steps:

1) signals collected by antennas are digitized right after amplification,

2) a temporal fast Fourier transform (FFT) is performed in order to split them into frequency subbands,

3) a spatial FFT is performed on each subband set,

4) the square modulus of the FFT result is computed,

5) an inverse spatial FFT is performed to recover the visibilities.

There are conceptual similarities with the bolometric interferometer concepts, since in this instrument, steps two and three are performed in an analogical way by the beam combiner and the bolometers, respectively. Because antennas have to be located on a grid in order for the FFTs to be performed, an omniscope will possess many redundant baselines, and Liu et al. (2010) have shown how this allows self-calibrating the complex gains of the antennas. In both cases (standard radio-interferometer or omniscope), the aim is to calibrate the complex gains of the antennas and there is actually a mathematical trick to get a linear system of equations from which these gains can be obtained. The self-calibration procedure explained in this paper is inspired by methods used in the paper of Liu et al. (2010) even if the design of the QUBIC instrument is different.

This paper is organized as follows. Section 2 introduces the self-calibration method with the Jones matrix formalism and the Mueller matrix formalism for radio-interferometry and the omniscope. We show in Sect. 3 how the procedure can be applied to the QUBIC bolometric interferometer, and finally we describe the possible self-calibration algorithm and its results.

\section{General principle}

\subsection{Instrumental systematics modelization with Jones matrices}

In this section, we use the notation proposed by (O'Dea et al. 2007). With the electric field of an incident radiation at a frequency $\phi$ defined as $\mathbb{R}\left[\vec{E} \mathrm{e}^{-\mathrm{i} \phi t}\right]$, and choosing two basis vectors $\overrightarrow{e_{x}}$ and $\overrightarrow{e_{y}}$ orthogonal to the direction of propagation $\vec{k}$, all the statistical information is encoded in the coherence matrix $\mathbf{C}$

$$
\begin{aligned}
\mathbf{C}=\left(\begin{array}{ll}
C_{x x} & C_{x y} \\
C_{x y}^{*} & C_{y y}
\end{array}\right) & =\left(\begin{array}{cc}
\left\langle\left|E_{x}\right|^{2}\right\rangle & \left\langle E_{x} E_{y}^{*}\right\rangle \\
\left\langle E_{x}^{*} E_{y}\right\rangle & \left\langle\left|E_{y}\right|^{2}\right\rangle
\end{array}\right) \\
& =\frac{1}{2}\left(\begin{array}{cc}
I+Q & U-\mathrm{i} V \\
U+\mathrm{i} V & I-Q
\end{array}\right),
\end{aligned}
$$

where $E_{x}, E_{y}$ are complex amplitudes of the transverse electric field $\vec{E}$ and $I, Q, U$, and $V$ are the Stokes parameters.

The propagation of an incident radiation $\vec{E}$ through a receiver can be described by a Jones matrix $\mathbf{J}$ such that the electric field after passing through the receiver $\vec{E}_{r}$ is

$\overrightarrow{E_{r}}=\mathbf{J} \vec{E}$ where the Jones matrix $\mathbf{J}$ is a $2 \times 2$ complex matrix. It describes how the instrument linearly transforms the two-dimensional vector representing the incoming radiation field $\vec{E}$ into the twodimensional vector of the outgoing field $\vec{E}_{r}$.

For an instrument with several components, the Jones matrix is the product of the Jones matrices for each component. For example, the ideal Jones matrix for an instrument in which the incident radiation passing through a rotating half-wave plate before propagating through the horns is

$$
\begin{array}{r}
\mathbf{J}_{\mathrm{hwp}}=\mathbf{J}_{\mathrm{rot}}^{\mathrm{T}} \mathbf{J}_{\mathrm{hwp}} \mathbf{J}_{\mathrm{rot}} \\
=\left(\begin{array}{cc}
\cos (\omega t) & -\sin (\omega t) \\
\sin (\omega t) & \cos (\omega t)
\end{array}\right)\left(\begin{array}{cc}
1 & 0 \\
0 & -1
\end{array}\right)\left(\begin{array}{cc}
\cos (\omega t) & \sin (\omega t) \\
-\sin (\omega t) & \cos (\omega t)
\end{array}\right) \\
=\left(\begin{array}{cc}
\cos (2 \omega t) & \sin (2 \omega t) \\
\sin (2 \omega t) & -\cos (2 \omega t)
\end{array}\right),
\end{array}
$$

where $\omega$ is the angular velocity of the half-wave plate, $\mathbf{J}_{\text {rot }}$ the rotation matrix and $\mathbf{J}_{\mathrm{hwp}}$ the ideal Jones matrix of the half-wave plate.

After passing through the receiver, ideal orthogonal linear detectors measure the power in two components

$$
\begin{aligned}
& S_{1}=\frac{1}{2}(I+Q \cos (4 \omega t)+U \sin (4 \omega t)) \\
& S_{2}=\frac{1}{2}(I-Q \cos (4 \omega t)-U \sin (4 \omega t)) .
\end{aligned}
$$

To model systematic errors within a polarization-sensitive interferometer, the Jones matrix can be described by introducing diagonal terms: the complex gain parameters $g_{x}$ and $g_{y}$ and nondiagonal terms: the complex coupling parameters $e_{x}$ and $e_{y}$ associated to the orthogonal polarizations

$\mathbf{J}=\left(\begin{array}{cc}1-g_{x} & e_{x} \\ e_{y} & 1-g_{y}\end{array}\right)$.

Systematic errors arising from the half-wave plate and from the square horn array can be modeled by

1. a Jones matrix for the half-wave plate

$$
\mathbf{J}_{\mathrm{hwp}}=\left(\begin{array}{cc}
1-h_{x} & \xi_{x} \\
\xi_{y} & -\left(1+h_{y}\right)
\end{array}\right),
$$

2. a Jones matrix for the horn $i$ with $1 \leqslant i \leqslant n_{h}$

$$
\mathbf{J}_{\mathrm{horn}, i}=\left(\begin{array}{cc}
1-g_{x, i} & e_{x, i} \\
e_{y, i} & 1-g_{y, i}
\end{array}\right) .
$$

The electric field $\vec{E}$ propagated through the half-wave plate and the horn $i$ becomes

$\vec{E}_{i}=\mathbf{J}_{\mathrm{horn}, i} \mathbf{J}_{\text {rot }}^{\mathrm{T}} \mathbf{J}_{\mathrm{hwp}} \mathbf{J}_{\mathrm{rot}} \vec{E}$.

\subsection{Instrumental systematics modelisation with Mueller matrix}

The Jones matrix expresses the transformation of the electric field in the $x$ and $y$-directions and the Mueller matrix describes how the different polarization states transform. The Jones matrix is a $2 \times 2$ matrix, whereas the Mueller matrix is a $4 \times 4$ matrix. The $4 \times 4$ Mueller matrix can thus be written as the direct product of the $2 \times 2$ Jones matrices. 
We calculate the tensor product of the outgoing field given by Eq. (2)

$$
\vec{E}_{r}^{*} \otimes \vec{E}_{r}=\mathbf{J} \vec{E} \otimes \mathbf{J}^{*} \vec{E}^{*}
$$

In general, the direct product $\left\langle\vec{E} \otimes \vec{E}^{*}\right\rangle$ gives the vector $\vec{C}$ defined as

$\vec{C}=\left[\begin{array}{l}C_{x x} \\ C_{x y} \\ C_{y x} \\ C_{y y}\end{array}\right]=\left\langle\vec{E} \otimes \vec{E}^{*}\right\rangle=\left\langle\left[\begin{array}{c}E_{x} \\ E_{y}\end{array}\right] \otimes\left[\begin{array}{c}E_{x}^{*} \\ E_{y}^{*}\end{array}\right]\right\rangle=\left[\begin{array}{c}\left\langle E_{x} E_{x}^{*}\right\rangle \\ \left\langle E_{x} E_{y}^{*}\right\rangle \\ \left\langle E_{y} E_{x}^{*}\right\rangle \\ \left\langle E_{y} E_{y}^{*}\right\rangle\end{array}\right]$

With Eq. (8), one can write the transmission of the electric field through an instrument described by its Jones matrix using the vector $\vec{C}$ of this electric field using the matrix direct product ${ }^{1}$

$\left.\vec{C}_{r}=\left\langle\vec{J} \vec{E} \otimes \mathbf{J}^{{ }^{*}} \vec{E}^{*}\right\rangle=\left\langle\mathbf{J} \otimes \mathbf{J}^{*}\right\rangle \vec{E} \otimes \vec{E}^{*}\right\rangle=\left\langle\mathbf{J} \otimes \mathbf{J}^{*}\right\rangle \vec{C}$

Accordingly Eq. (1), the polarization state of this electric field can be described by the Stokes vector $\vec{S}$ defined by

$\vec{S}=\left[\begin{array}{c}I \\ Q \\ U \\ V\end{array}\right]=\left[\begin{array}{c}C_{x x}+C_{y y} \\ C_{x x}-C_{y y} \\ C_{x y}+C_{y x} \\ \mathrm{i}\left(C_{x y}-C_{y x}\right)\end{array}\right]$,

where $I, Q, U$, and $V$ are the Stokes parameters.

One can obtain the expression of the Stokes vector from the vector $\vec{C}$ defined in Eq. (9)

$\vec{S}=\mathbf{A C}$,

where

$$
\mathbf{A}=\left[\begin{array}{cccc}
1 & 0 & 0 & 1 \\
1 & 0 & 0 & -1 \\
0 & 1 & 1 & 0 \\
0 & \mathrm{i} & -\mathrm{i} & 0
\end{array}\right]
$$

Substituting Eq. (12) into Eq. (10), it follows that the outgoing Stokes vector $\vec{S}_{r}$ can be written as

$\vec{S}_{r}=\mathbf{A}\left(\mathbf{J} \otimes \mathbf{J}^{*}\right) \mathbf{A}^{-1} \vec{S}=\mathbf{M} \vec{S}$

where $\mathbf{M}=\mathbf{A}\left(\mathbf{J} \otimes \mathbf{J}^{*}\right) \mathbf{A}^{-1}$ is the Mueller matrix that describes how the Stokes parameters transform.

\subsection{Polarized measurement equation with Mueller formalism}

A polarization-sensitive interferometer measures the complex Stokes visibilities from all baselines defined by the horns $i$ and $j$ in an array of receivers

$V_{i j}=\left(\begin{array}{c}V_{i j}^{I} \\ V_{i j}^{Q} \\ V_{i j}^{U} \\ V_{i j}^{V}\end{array}\right)$.

1 If $\mathbf{A}$ and $\mathbf{B}$ are $2 \times 2$ matrices defined as $A=\left(\begin{array}{ll}A_{11} & A_{12} \\ A_{21} & A_{22}\end{array}\right)$ and $B=$ $\left(\begin{array}{ll}B_{11} & B_{12} \\ B_{21} & B_{22}\end{array}\right)$, the matrix direct product of $\mathbf{A}$ and $\mathbf{B}$ is

$$
\mathbf{A} \otimes \mathbf{B}=\left(\begin{array}{ll}
A_{11} \mathbf{B} & A_{12} \mathbf{B} \\
A_{21} \mathbf{B} & A_{22} \mathbf{B}
\end{array}\right)=\left(\begin{array}{llll}
A_{11} B_{11} & A_{11} B_{12} & A_{12} B_{11} & A_{12} B_{12} \\
A_{11} B_{21} & A_{11} B_{22} & A_{12} B_{21} & A_{12} B_{22} \\
A_{21} B_{11} & A_{21} B_{12} & A_{22} B_{11} & A_{22} B_{12} \\
A_{21} B_{21} & A_{21} B_{22} & A_{22} B_{21} & A_{22} B_{22}
\end{array}\right) .
$$

These vectors could reduce to a scalar or a vector with 2,3 , or 4 elements depending on the Stokes parameters the instrument is sensitive to. One can define $a=1,2,3,4$ as the number of Stokes parameters the instrument allows to be measured.

The $n_{\mathrm{h}}\left(n_{\mathrm{h}}-1\right) / 2$ baselines of the interferometer can be classified into $n_{\neq}$sets $s_{\beta}$ of redundant baselines (same length, same direction) indexed by $\beta$. In the absence of systematic errors, the redundant visibilities should have exactly the same values

$\forall\{i, j\} \epsilon s_{\beta}, V_{i j}=V_{\beta}$.

For a real instrument, however, redundant visibilites $\bar{V}_{i j}$ will not have exactly the same values because of systematic errors and statistical (photon) noise, and one can write the following system of $a \times n_{\mathrm{h}}\left(n_{\mathrm{h}}-1\right) / 2$ complex equations

$\bar{V}_{i j}=\mathbf{M}_{i j} \cdot V_{\beta}+n_{i j}$

where $n_{i j}$ are statistical noise terms and where $\mathbf{M}_{i j}$ are a kind of complex Mueller matrices that reduce to the identity matrix for a perfect instrument:

$\mathbf{M}_{i j}=\left(\begin{array}{llll}M_{i j}^{I I} & M_{i j}^{I Q} & M_{i j}^{I U} & M_{i j}^{I V} \\ M_{i j}^{Q I} & M_{i j}^{Q Q} & M_{i j}^{Q U} & M_{i j}^{Q V} \\ M_{i j}^{U I} & M_{i j}^{U Q} & M_{i j}^{U U} & M_{i j}^{U V} \\ M_{i j}^{V I} & M_{i j}^{V Q} & M_{i j}^{V U} & M_{i j}^{V V}\end{array}\right)$.

The elements of these matrices are not independent and can be expressed in terms of the diagonal and the nondiagonal terms of the Jones matrix.

The first order Mueller matrix for a polarization sensitive experiment is

$M_{i j}=\frac{1}{2}\left(\begin{array}{ll}A & B \\ C & D\end{array}\right)$

with

$A=\left(\begin{array}{cc}2+g_{x, j}^{*}+g_{y, j}^{*}+g_{x, i}+g_{y, i} & g_{x, j}^{*}-g_{y, j}^{*}+g_{x, i}-g_{y, i} \\ g_{x, j}^{*}-g_{y, j}^{*}+g_{x, i}-g_{y, i} & 2+g_{x, j}^{*}+g_{y, j}^{*}+g_{x, i}+g_{y, i}\end{array}\right)$

$B=\left(\begin{array}{l}e_{x, j}^{*}+e_{y, j}^{*}+e_{x, i}+e_{y, i}-\mathrm{i}\left(e_{x, j}^{*}-e_{y, j}^{*}-e_{x, i}+e_{y, i}\right) \\ e_{x, j}^{*}-e_{y, j}^{*}+e_{x, i}-e_{y, i}-\mathrm{i}\left(e_{x, j}^{*}+e_{y, j}^{*}-e_{x, i}-e_{y, i}\right)\end{array}\right)$

$C=\left(\begin{array}{cc}e_{x, j}^{*}+e_{y, j}^{*}+e_{x, i}+e_{y, i} & -e_{x, j}^{*}+e_{y, j}^{*}-e_{x, i}+e_{y, i} \\ -\mathrm{i}\left(e_{x, j}^{*}-e_{y, j}^{*}-e_{x, i}+e_{y, i}\right) & \mathrm{i}\left(e_{x, j}^{*}+e_{y, j}^{*}-e_{x, i}-e_{y, i}\right)\end{array}\right)$

$D=\left(\begin{array}{lc}2+g_{x, j}^{*}+g_{y, j}^{*}+g_{x, i}+g_{y, i} & \mathrm{i}\left(g_{x, j}^{*}-g_{y, j}^{*}-g_{x, i}+g_{y, i}\right) \\ -\mathrm{i}\left(g_{x, j}^{*}-g_{y, j}^{*}+g_{x, i}+g_{y, i}\right) & 2+g_{x, j}^{*}+g_{y, j}^{*}+g_{x, i}+g_{y, i}\end{array}\right)$.

\section{Application to the QUBIC bolometric interferometer}

\subsection{Observables in bolometric interferometry}

In this section, we derive the expression for the power received in the focal plane in the case of bolometric interferometry. The bolometric interferometer proposed with the QUBIC instrument (the QUBIC collaboration 2010) is the millimetric equivalent of the first interferometer dedicated to astronomy: the Fizeau interferometer - see Fig. 1 for the design of the QUBIC instrument. 


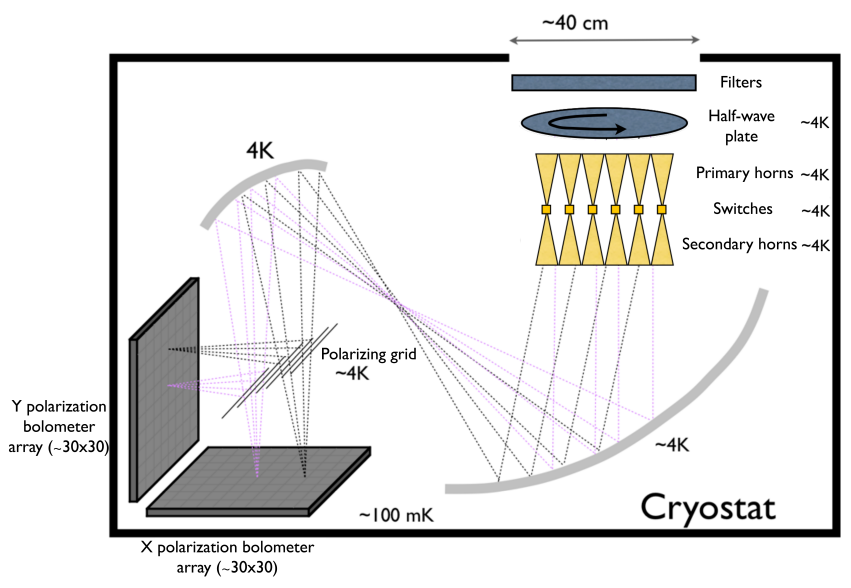

Fig. 1. Design of the QUBIC instrument.

The receptors are two arrays of $n_{\mathrm{h}}$ horns: the primary and secondary horns back-to-back on a square grid behind the optical window of a cryostat. Filters and switches are placed in front and between the horn array. The switches will be only used during the calibration phase. The polarization of the incoming field is modulated using a half-wave plate located before the primary horns. This location of the half-wave plate avoids a leakage from the Stokes parameter $I$ to the Stokes parameters $Q$ and $U$ if the half-wave plate has no inhomogeneities.

Signals are correlated together using an optical combiner. The interference fringe patterns arising from all pairs of horns, with a given angle, are focused to a single point on the focal plane. Finally, a polarizing grid splits the signal into $x$ and $y$-polarizations, each being focused on a focal plane equipped with bolometers. These bolometers measure a linear combination of the Stokes parameters modulated by the rotating halfwave plate.

With an interferometer, the correlation between two receivers allows for direct access to the Fourier modes (visibilities) of the Stokes parameters $I, Q$, and $U$. In the case of a bolometric interferometer, the observable is the superposition of the fringes formed by the sky electric field passing through a large number of back-to-back horns and then focused on the detector plane array. The image on the focal plane of the optical combiner is the synthesized image, because only specific Fourier modes are selected by the receiving horns array. A bolometric interferometer is therefore a synthesized imager whose beam is the synthesized beam formed by the array of receiving horns.

In the following section, we begin by defining the formalism for the QUBIC instrument in an ideal case, that is to say, without systematics. The electric field $E^{\eta}\left(\hat{n}_{\mathrm{p}}\right)$ passing through a half-wave plate that rotates at angular speed $\omega$ and modulates the two orthogonal polarizations $\eta \in\{x, y\}$ becomes, as a function of the observed direction $\hat{n}_{\mathrm{p}}$,

$\left(\begin{array}{l}E^{\prime x}\left(\hat{n}_{\mathrm{p}}\right) \\ E^{\prime y}\left(\hat{n}_{\mathrm{p}}\right)\end{array}\right)=\left(\begin{array}{cc}\cos (2 \omega t) & \sin (2 \omega t) \\ \sin (2 \omega t) & -\cos (2 \omega t)\end{array}\right)\left(\begin{array}{l}E^{x}\left(\hat{n}_{\mathrm{p}}\right) \\ E^{y}\left(\hat{n}_{\mathrm{p}}\right)\end{array}\right)$.

For an ideal instrument, the electric field, after passing through the half-wave plate, collected by one horn $i$ located at $\vec{x}_{i}$ for the polarization $\eta \in\{x, y\}$ when all the primary horns are looking at the same radiation field $E^{\eta}\left(\hat{n}_{\mathrm{p}}\right)$ as a function of the observed direction $\hat{n}_{\mathrm{p}}$ and reaching the bolometer $q$ at the direction $\hat{d}_{q}$ viewed from the optical center of the beam combiner is

$\left(\begin{array}{c}s_{i q}^{x}\left(\hat{n}_{\mathrm{p}}\right) \\ s_{i q}^{y}\left(\hat{n}_{\mathrm{p}}\right)\end{array}\right)=\alpha_{i q} \bullet \beta_{i}\left(\hat{n}_{\mathrm{p}}\right) \bullet\left(\begin{array}{l}E^{\prime x}\left(\hat{n}_{\mathrm{p}}\right) \\ E^{\prime y}\left(\hat{n}_{\mathrm{p}}\right)\end{array}\right)$ where $\alpha_{i q} \bullet \beta_{i}\left(\hat{n}_{\mathrm{p}}\right)$ is the product matrix of the matrices $\alpha_{i q}$ and $\beta_{i}\left(\hat{n}_{\mathrm{p}}\right)$.

We have introduced the matrices $\beta_{i}\left(\hat{n}_{\mathrm{p}}\right)$ and $\alpha_{i q}$ which characterize completely the instrument. A matrix $\beta_{i}\left(\hat{n}_{\mathrm{p}}\right)$ is defined for each channel of pointings (p) and horns (i) and includes the primary beam $B_{\text {prim }, i}^{\eta}\left(\hat{n}_{\mathrm{p}}\right)$, the horn position $\vec{x}_{i}$ and the direction of pointing $\hat{n}_{\mathrm{p}}$

$\beta_{i}^{\text {ideal }}\left(\hat{n}_{\mathrm{p}}\right)=\left(\begin{array}{cc}\beta_{i}^{x}\left(\hat{n}_{\mathrm{p}}\right) & 0 \\ 0 & \beta_{i}^{y}\left(\hat{n}_{\mathrm{p}}\right)\end{array}\right)$

with

$\beta_{i}^{x}\left(\hat{n}_{\mathrm{p}}\right)=B_{\mathrm{prim}, i}^{x}\left(\hat{n}_{\mathrm{p}}\right) \exp \left(\mathrm{i} 2 \pi \frac{\vec{x}_{i} \cdot \hat{n}_{\mathrm{p}}}{\lambda}\right)$,

and

$\beta_{i}^{y}\left(\hat{n}_{\mathrm{p}}\right)=B_{\mathrm{prim}, i}^{y}\left(\hat{n}_{\mathrm{p}}\right) \exp \left(\mathrm{i} 2 \pi \frac{\vec{x}_{i} \cdot \hat{n}_{\mathrm{p}}}{\lambda}\right)$.

A matrix $\alpha_{i q}$ is defined for each channel of horns (i) and bolometers (q) and includes the geometrical phases induced by the beam combiner, the beams of the secondary horns $B_{\mathrm{sec}}^{\eta}\left(\hat{d}_{q}\right)$, and the gain $g_{q}$ of the bolometer ${ }^{2}$

$\alpha_{i q}^{\text {ideal }}=\left(\begin{array}{cc}\alpha_{i q}^{x} & 0 \\ 0 & \alpha_{i q}^{y}\end{array}\right)$

with

$\alpha_{i q}^{x}=g_{q} \int B_{\mathrm{sec}}^{x}\left(\hat{d}_{q}\right) \exp \left[\mathrm{i} 2 \pi \frac{\vec{x}_{i} \cdot \hat{d}_{q}}{\lambda}\right] J(v) \Theta\left(\hat{d}-\hat{d}_{q}\right) \mathrm{d} v \mathrm{~d} \hat{d}_{q}$,

and

$\alpha_{i q}^{y}=g_{q} \int B_{\mathrm{sec}}^{y}\left(\hat{d}_{q}\right) \exp \left[\mathrm{i} 2 \pi \frac{\vec{x}_{i} \cdot \hat{d}_{q}}{\lambda}\right] J(v) \Theta\left(\hat{d}-\hat{d}_{q}\right) \mathrm{d} v \mathrm{~d} \hat{d}_{q}$.

The detector direction as viewed from the optical center of the optical combiner is given by the unit vector $\hat{d}_{q}$, and $\lambda$ is the wavelength of the instrument. The integrations are over the surface of each individual bolometer modeled with the top-hat like function $\Theta\left(\hat{d}-\hat{d}_{q}\right)^{3}$, and on bandwidths of the instrument $J(v)$ with $v$ the frequency.

We assume that there is no cross-polarization because the matrices $\beta_{i}\left(\hat{n}_{\mathrm{p}}\right)$ and $\alpha_{i q}$ are written for an ideal case so that there is no dependence on the direction of polarization. The power measured by one polarized bolometer located at $\hat{d}_{q}$ in the focal plane of the beam combiner is then

$S_{q p}^{\eta}=\int\left|\sum_{i} s_{i q}^{\eta}\left(\hat{n}_{\mathrm{p}}\right)\right|^{2} \mathrm{~d} \hat{n}_{\mathrm{p}}$

where $s_{i q}^{\eta}\left(\hat{n}_{\mathrm{p}}\right)$ is given in Eq. (24).

2 The expressions written here are only valid in the quasi-normal incidence regime. This is not an issue, because in the next section we see that we reconstruct the actual value of $\alpha_{i q}^{\eta}$ regardless of its exact expression.

3 In the case of the QUBIC instrument, the wavelength is within an order of magnitude of the detector size, consequently the field rather than the flux is being integrated over the bolometer area to take the acceptance diffraction-limited beam into account. 
Without systematics, Eq. (31) can be written as

$S_{q p}^{\eta}=\int\left|E^{\prime \eta}\left(\hat{n}_{\mathrm{p}}\right)\right|^{2} B_{q, s}^{\eta}\left(\hat{n}_{\mathrm{p}}\right) \mathrm{d} \hat{n}_{\mathrm{p}}$

where the synthesized beam $B_{q, s}^{\eta}\left(\hat{n}_{\mathrm{p}}\right)$ for the detector $q$ is formed by the arrangement of the primary horns array as

$B_{q, s}^{\eta}\left(\hat{n}_{\mathrm{p}}\right)=\sum_{i, j} \alpha_{i q}^{\eta} \alpha_{j q}^{* \eta} \beta_{i}^{\eta}\left(\hat{n}_{\mathrm{p}}\right) \beta_{j}^{* \eta}\left(\hat{n}_{\mathrm{p}}\right)$

The synthesized beam depends on the sky direction $\left(\hat{n}_{\mathrm{p}}\right)$, so the synthesized image is the convolution of the sky and of the electric field through the synthesized beam.

One can rewrite Eq. (31) to exhibit the modulation of the polarization induced by the half-wave plate as

$S_{q p}^{\eta}(t)=S_{q p}^{I}+\epsilon^{\eta} \cos (4 \pi \omega t) S_{q p}^{Q}+\epsilon^{\eta} \sin (4 \pi \omega t) S_{q p}^{U}$

where $\epsilon^{x}=1$ for the polarization $x, \epsilon^{y}=-1$ for the polarization $y$, and where $S_{q p}^{X}$ are the synthesized images on the focal plane for each Stokes parameter $X=\{I, Q, U\}$. The cosine and sine coefficients come from the modulation induced by the rotating half-wave plate.

Systematic effects arising at any level of the detection can be modeled by associating a Jones matrix to each horn i $\mathbf{J}_{\text {horn, } i}$ and a Jones matrix of the half-wave plate $\mathbf{J}_{\mathrm{hwp}}$. They can be introduced as defined in Eq. (7), and Eq. (24) becomes

$\left(\begin{array}{c}s_{i q}^{x}\left(\hat{n}_{\mathrm{p}}\right) \\ s_{i q}^{y}\left(\hat{n}_{\mathrm{p}}\right)\end{array}\right)=\alpha_{i q} \bullet \beta_{i}\left(\hat{n}_{\mathrm{p}}\right) \bullet \mathbf{J}_{\mathrm{horn}, i} \bullet \mathbf{J}_{\text {rot }}^{\mathrm{T}} \bullet \mathbf{J}_{\mathrm{hwp}} \bullet \mathbf{J}_{\mathrm{rot}} \bullet\left(\begin{array}{l}E^{x}\left(\hat{n}_{\mathrm{p}}\right) \\ E^{y}\left(\hat{n}_{\mathrm{p}}\right)\end{array}\right)$

where $E^{\eta}\left(\hat{n}_{\mathrm{p}}\right)$ is the incident electric field, $\mathbf{J}_{\text {horn, } i}$ the Jones matrix of the horn $i, \mathbf{J}_{\mathrm{hwp}}$ the Jones matrix of the half-wave plate, and $\mathbf{J}_{\text {rot }}$ the rotation matrix induced by the rotation of the halfwave plate.

\subsection{Self-calibration procedure}

During the self-calibration mode, which is distinct from the ordinary data-taking mode, the instrument scans a polarized source and measures the $n_{\mathrm{h}}\left(n_{\mathrm{h}}-1\right) / 2$ synthesized images one by one from all baselines or only a fraction of them. In the QUBIC design, this can be achieved using switches located between the back-to-back horns. The switches are used as shutters that are operated independently for all channels and are only required during the calibration phase. One can modulate on/off a single pair of horns while leaving all the others open in order to access the synthesized images measured by each pair of horns alone. This procedure requires the knowledge of the individual primary beams of each horn. The maps of the primary beams can be obtained independently through scanning an external unpolarized source.

By repeating this with all baselines, all bolometers, and all directions of pointing, one can construct a system of equations whose unknowns are

1. the complex coefficients $\alpha_{i q}^{\eta}$, defined for each horn $i$, each bolometer $q$, and for each polarization $\eta$ which correspond to $4 n_{\mathrm{h}} n_{q}$ parameters,

2. the horn location $\vec{x}_{i}\left(2 n_{\mathrm{h}}\right.$ parameters $)$,

3 . the direction of pointing $\hat{n}_{\mathrm{p}}\left(2 n_{\mathrm{p}}\right.$ parameters $)$,

4. the complex horns systematic effects $g_{x, i}, g_{y, i}, e_{x, i}, e_{y, i}$ defined for each horn $i$ and for each polarization $\eta\left(8 n_{h}\right.$ parameters),

5. the complex half-wave plate systematic effects $h_{x}, h_{y}, \xi_{x}, \xi_{y}$ (8 parameters).
For an instrument with $n_{\mathrm{h}}$ horns, $n_{q}$ bolometers and for a scan of $n_{\mathrm{p}}$ pointings, the number of unknowns is

$n_{u}=4 \times n_{\mathrm{h}} \times n_{q}+2 \times n_{\mathrm{h}}+2 \times n_{\mathrm{p}}+8 \times n_{\mathrm{h}}+8$.

During the self-calibration procedure, the number of constraints is given by the measurements, i.e. the synthesized images. One has the $n_{\mathrm{h}}\left(n_{\mathrm{h}}-1\right) / 2$ measured synthesized images for each bolometer $n_{q}$, each pointing $n_{\mathrm{p}}$, each Stokes parameter, and the two focal planes. The number of constraints is given by

$n_{\mathrm{c}}=6 \times n_{\mathrm{h}} \times\left(n_{\mathrm{h}}-1\right) / 2 \times n_{q} \times n_{\mathrm{p}}$.

The problem becomes easily overdetermined: for an instrument with $n_{\mathrm{h}}=9, n_{q}=4$, and $n_{\mathrm{p}}=10$, the number of constraints is 8640 and the number of unknowns is 262 . It can be solved with a least squares algorithm.

The first module of the QUBIC instrument will consist of 400 primary horns and two 1024 element bolometer arrays. The number of constraints could be reduced if the self-calibration is performed not on the $n_{\mathrm{h}}\left(n_{\mathrm{h}}-1\right) / 2$ baselines but on a fraction of baselines. This is shown in the following.

Closing all the switches except two would actually dramatically change the thermal load on the cryostat, which could affect the bolometric measurements. Fortunately, there is a trick explained in Appendix A that allows $S_{i j p q}^{\eta}\left(\hat{d}_{q}\right)$ to be indirectly measured while minimally changing the thermal load. One can show that

$S_{i j p q}^{\eta}\left(\hat{d}_{q}\right)=\bar{C}_{i p q}^{\eta}\left(\hat{d}_{q}\right)+\bar{C}_{j p q}^{\eta}\left(\hat{d}_{q}\right)-2 \bar{S}_{i j p q}^{\eta}\left(\hat{d}_{q}\right)$

where $\bar{S}_{i j p q}^{\eta}\left(\hat{d}_{q}\right)$ is the quantity measured by a bolometer $q$ when all the switches are open except the $i$ and $j$, and $\bar{C}_{i p q}^{\eta}\left(\hat{d}_{q}\right)$, $\bar{C}_{j p q}^{\eta}\left(\hat{d}_{q}\right)$ are the powers measured when all the switches are open except respectively $i$ or $j$. Measuring these three terms therefore allows measuring $S_{i j p q}^{\eta}\left(\hat{d}_{q}\right)$ while keeping the thermal load almost constant. However, this also increases the noise. The noise on each term is therefore

$$
\begin{aligned}
\Delta S_{i j p q}^{2} & =\Delta \bar{C}_{i p q}^{2}+\Delta \bar{C}_{j p q}^{2}+4 \Delta \bar{S}_{i j p q}^{2} \\
& =2\left(n_{\mathrm{h}}-1\right) N E T^{2}+4\left(n_{\mathrm{h}}-2\right) N E T^{2} \\
& =\left(6 n_{\mathrm{h}}-10\right) N E T^{2}
\end{aligned}
$$

where NET is the noise equivalent temperature of the bolometers, and $T$ the temperature of the $100 \%$ polarized source.

\subsection{Numerical simulation}

We have numerically implemented the method to check if the nonlinear system could be solved. We generate the instrument with a set of ideal parameters (horn locations, directions of pointing, primary and secondary beams, detector locations, etc.), and a set of parameters randomly corrupted by systematic errors (horn location errors, pointing errors, assymetries of beams, bolometer location errors, diagonal and nondiagonal terms of the Jones matrices, etc.). The widths of the random deviation of all corrupted parameters around their ideal value are given in Table 1. These values of corruption are independent Gaussian errors added to each parameter and each range of error is fixed according to the tolerance we impose on components.

The primary beams $B_{\text {prim }, i}^{\eta}\left(\hat{n}_{\mathrm{p}}\right)$ are assumed to be known perfectly so their values are not varied in the simulation; however, the matrix $\beta_{i}^{\eta}\left(\hat{n}_{\mathrm{p}}\right)$ varies as the horn locations $\vec{x}_{i}$ and the pointing 
Table 1. Range for systematic errors for each parameter.

\begin{tabular}{lr}
\hline \hline Error source & Gaussian level \\
\hline Pointing uncertainty $\hat{n}_{\mathrm{p}}$ & $1[\mathrm{deg}]$ \\
Horn location error $\vec{x}_{i}$ & $100[\mu \mathrm{m}]$ \\
Detector location error & $1[\mu \mathrm{m}]$ \\
Bolometer gain $g_{q}$ & 0.01 \\
Primary beam error $B_{\mathrm{prim}, i}^{\eta}\left(\hat{n}_{\mathrm{p}}\right)$ & 0.01 \\
Secondary beam error $B_{\mathrm{sec}}^{\eta}\left(\hat{d}_{q}\right)$ & 0.01 \\
Horn systematics $g_{\eta, i}$ & 0.0001 \\
Horn systematics $e_{\eta, i}$ & 0.0001 \\
Half-wave plate systematics $h_{\eta}$ & 0.01 \\
Half-wave plate systematics $\xi_{\eta}$ & 0.01 \\
\hline
\end{tabular}

Notes. The systematics are independent Gaussian errors added to each parameter. Each range of error is fixed according to the tolerance we impose on components.

directions $\hat{n}_{\mathrm{p}}$ are unknowns of the system. In the simulation, the angular position of the half-wave plate is drawn at random. A random angular position of the half-wave plate is given for each measurement.

To get a solvable system, one must add some normalization constraints for the coefficients $\alpha_{i q}^{\eta}$ and $\beta_{i}^{\eta}\left(\hat{n}_{\mathrm{p}}\right)$, which do not change the modeling of systematic errors. They mean that the self-calibration only allows for relative calibration of these parameters. One can add

1. an absolute calibration of the global gain of the instrument, $\alpha_{00}^{\eta}=1$.

2. a convention on the phase of the $\alpha_{i q}^{\eta}$ coefficients, $\forall q, \arg \left(\alpha_{0 q}^{\eta}\right)=0$. A rotation of global phase $\phi_{q}$ applied to the coefficients $\alpha_{i q}^{\eta}$ for one bolometer $q$ does not modify Eqs. (24) and (31) and therefore the observations.

3. a convention on the primary beams, $\left.\forall i, \beta_{i}^{\eta}(\hat{0})\right)=1$. Multiplying the $\alpha_{i q}^{\eta}$ coefficients by a term defined as $c_{i} \mathrm{e}^{\phi_{i}}$ and dividing the $\beta_{i}^{\eta}\left(\hat{n}_{\mathrm{p}}\right)$ coefficients by the same term does not modify Eqs. (24) and (31) and therefore the observations.

4. a convention on the phase of the $\beta_{i}^{\eta}\left(\hat{n}_{\mathrm{p}}\right)$ coefficients, $\forall p, \arg \left(\beta_{0}^{\eta}\left(\hat{n}_{\mathrm{p}}\right)\right)=0$. A rotation of global phase $\phi\left(\hat{n}_{\mathrm{p}}\right)$ applied to the $\beta_{i}^{\eta}\left(\hat{n}_{\mathrm{p}}\right)$ coefficients for one pointing $\mathrm{p}$ does not modify Eqs. (24) and (31) and the observations.

5. an absolute calibration of the overall gain of the horns, $g_{x, 0}=$ 1. It means that the self-calibration procedure only allows a relative calibration of the gain terms.

We compute the corrupted synthesized images and add Gaussian statistic noise given by

$n_{\text {noise }}=\frac{N E T_{\mathrm{P}}}{\sqrt{t_{\mathrm{b}}} \sqrt{2}} \sqrt{6 n_{\mathrm{h}}-10}$

where the noise equivalent temperature of the bolometers NET is taken to be $300 \mu \mathrm{K} \mathrm{s}^{\frac{1}{2}}$, the temperature of the $100 \%$ polarized source is $T=100 \mathrm{~K}$, and the time spent on each baseline on the calibration mode is $t_{\mathrm{b}}=1 \mathrm{~s}$. The usual convention is to give the NET for unpolarized detectors, but it is convenient in our case to use quantities with polarization. In this case, the NET is given by $N E T=\frac{N E T_{\mathrm{P}}}{\sqrt{2}}$.

We solve the nonlinear system with a standard nonlinear least-squares method based on a Levenberg-Marquardt algorithm. The ideal coefficients (without systematic errors) are used as starting guess for the different parameters.

\subsection{Results}

To be able to have a large number of realizations, we run the simulation for an array of nine primary horns, nine bolometers, and ten pointings for 100 realizations. Figure 2 shows the result of the self-calibration simulation. The six plots are scatter plots of ideal vs. real synthesized images in red and of recovered vs. real synthesized images in blue for the $X$ and $Y$ focal planes and each Stokes parameter $I, Q$, and $U$. The synthesized images are computed with Eq. (31), the ideal synthesized images are the synthesized images without systematic effects, the real synthesized images are the simulated measurements, and the recovered synthesized images are computed with the output parameters of the self-calibration simulation. The six plots show the advantage of the self-calibration method.

This method allows access to the systematic effects of the horns and of the half-wave plate. It also allows calibrating the parameters $\alpha_{i q}^{\eta}$ and $\beta_{i}\left(\hat{n}_{\mathrm{p}}\right)$ that completely characterize the instrument for each channel of pointings, horns, and bolometers.

In running the simulation, one can find that the residual error on each output parameter will depend on the number of horns, bolometers, pointings, baselines per pointings, and on the time spent measuring each baseline. Adding more horns, pointings, bolometers, and baselines increases the mathematical constraints on a given measurement, it allows to form new baselines and adds redundancy on the horn array. Figure 3 shows that the residual diagonal term error of the Jones matrix of the half-wave plate improves as the number of baselines per pointing is higher. This result was obtained for a simulation with nine primary horns, nine bolometers and ten pointings for 40 realizations and for a time spent on each baseline $t_{\mathrm{b}}=1 \mathrm{~s}$. The baselines measured for each pointing are chosen randomly. Similar plots are obtained with the other parameters defined in Table 2.

One can put together these variables and define a power law that allows calculating the residual error on each parameter defined in Table 1

$\sigma_{\mathrm{c}} \sim c \times \frac{1}{n_{\mathrm{h}}^{\alpha}} \times \frac{1}{n_{q}^{\beta}} \times \frac{1}{n_{\mathrm{p}}^{\gamma}} \times \frac{1}{n_{\mathrm{bs}}^{\Phi}}$

with $c$ a constant and $\alpha, \beta, \gamma, \Phi$ the exponent of the number of horns $n_{\mathrm{h}}$, bolometers $n_{\mathrm{q}}$, pointings $n_{\mathrm{p}}$, and the percent of baselines per pointing $n_{\mathrm{bs}}$.

The values for each index are summarized in Table 2 for two different measuring times per baseline $t_{\mathrm{b}}=1$ and $t_{\mathrm{b}}=100 \mathrm{~s}$.

One can observe in Table 2 that the error on the different reconstructed parameters is better when the time spent on each baseline is longer. Figure 4 represents the residual half-wave plate gain error as a function of the time spent on each baseline during the calibration mode. It shows that the limitation of the accuracy achieved on the systematic parameters is given by the time spent on calibration mode for each baseline $t_{\mathrm{b}}$.

The first QUBIC module will contain 400 primary horns, or 79800 baselines; therefore, we need to spend about $22 \mathrm{~h}$ on calibration in order to measure all the baselines during one second. This lapse of time could, however, be much reduced with a small information loss if the self-calibration procedure was not performed on all baselines. The accuracy on the output parameters also depends on the number of baselines per measurement as illustrated in Fig. 3. It will be important to determine which is the most interesting strategy for the QUBIC instrument.

Using the law given by Eq. (40), one can extrapolate the result given in Table 2 to the residual error for the QUBIC instrument with 400 horns, $2 \times 1024$ bolometers, and 1000 pointings for two different measuring times per baseline $t_{\mathrm{b}}=1$ and 
M.-A. Bigot-Sazy et al.: Self-calibration: an efficient method to control systematic effects in bolometric interferometry
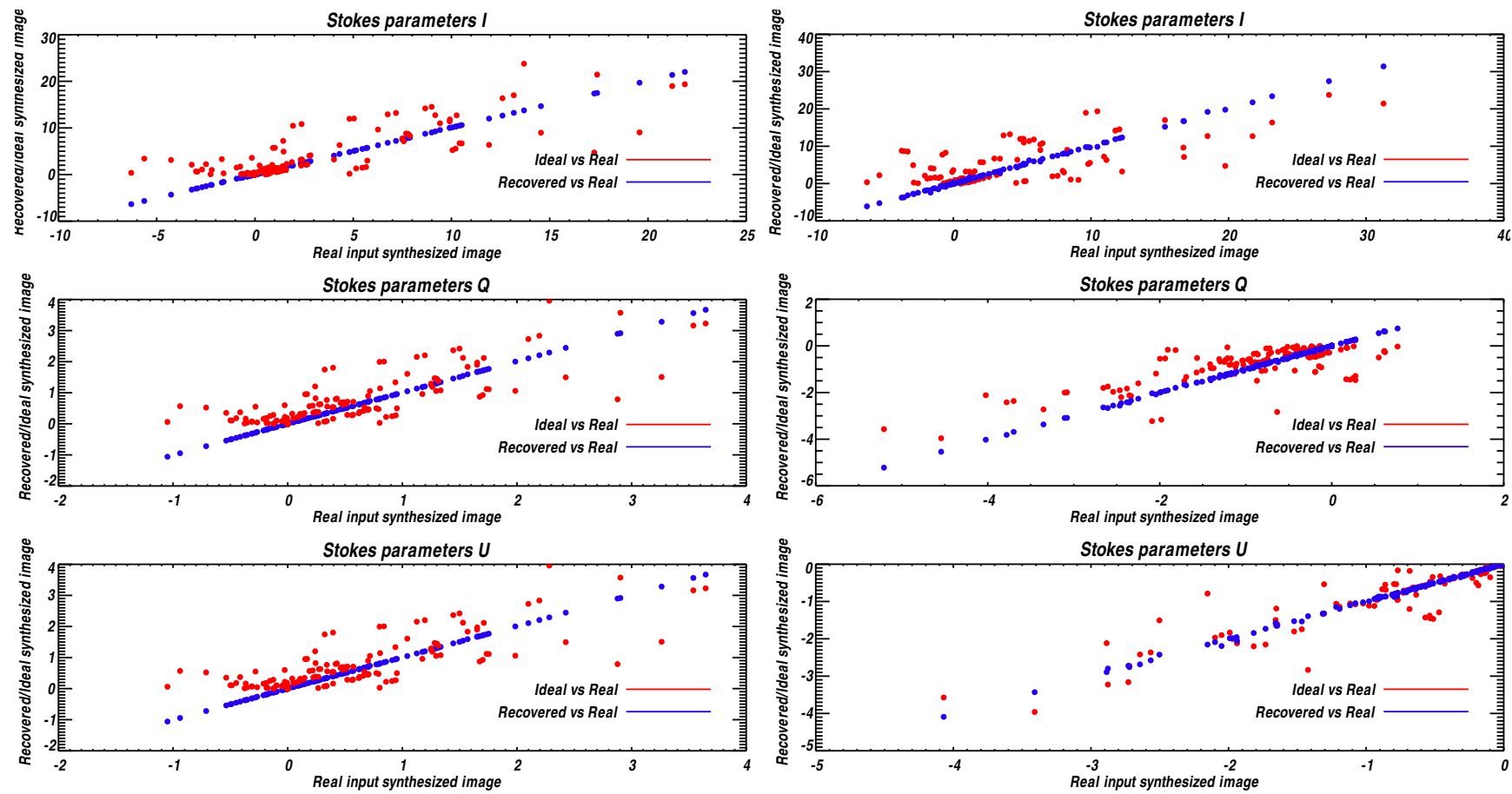

Fig. 2. Results of the self-calibration simulation for the synthesized images $S_{q p}^{I}, S_{q p}^{Q}$, and $S_{q p}^{U}$ for the $X$ focal plane on the right and $Y$ focal plane on the left for an instrument with nine primary horns, nine bolometers, and ten pointings for a time spent on calibration mode for each baseline of $t_{\mathrm{b}}=1 \mathrm{~s}$ and 100 realizations. These plots represent scatter plots of ideal vs. real synthesized image in red and of recovered vs. real in blue. The red plots show the corruption after adding the systematic effects defined in Table 1. The blue plots show that the corruption is solved after applying the self-calibration method.

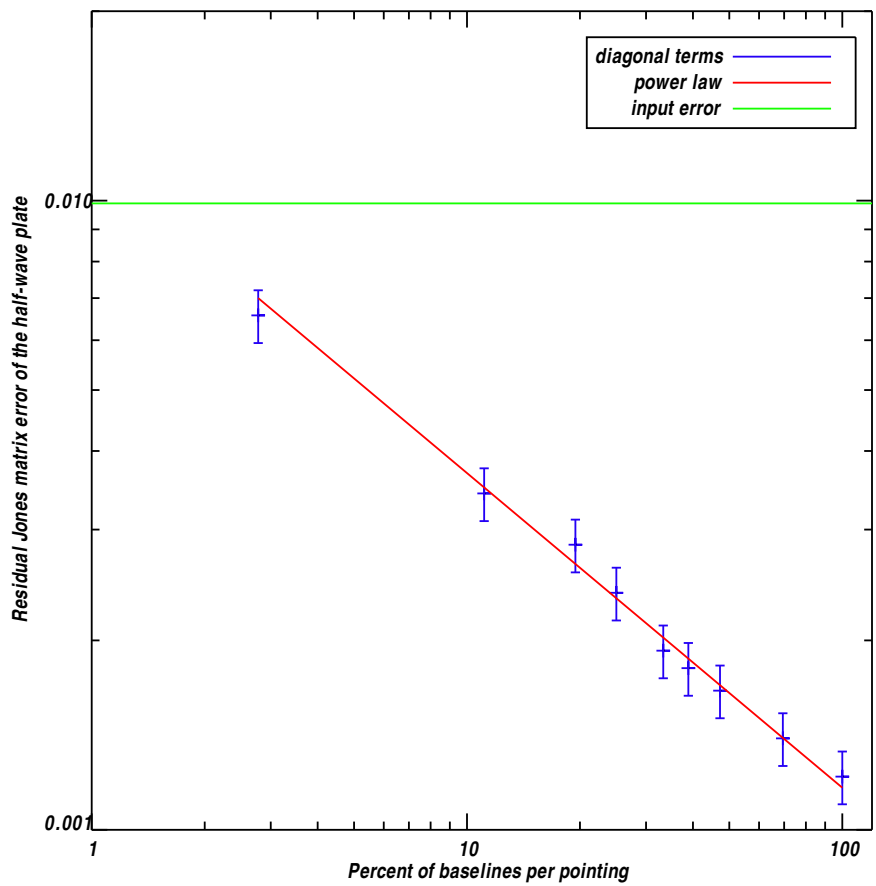

Fig. 3. Results of the self-calibration simulation for the diagonal terms of the Jones matrix of the half-wave plate and a time spent per baseline $t_{\mathrm{b}}=1 \mathrm{~s}$. This plot represents the residual error on these parameters as a function of the percent of baselines per pointing. The red line represents a power law of the shape $\sim \frac{c}{n_{\mathrm{b}}^{\Phi}}$ with $\mathrm{c}$ a constant, $n_{\mathrm{bs}}$ the number of baselines per pointing and $\Phi$ the index of the power law given in Table 2. This law gives the limit of the relative accuracy that can be achieved on the systematic parameters. The green line represents the input error on the systematic effects of the half-wave plate in the simulation given in Table 1. $t_{\mathrm{b}}=100 \mathrm{~s}$. The result is given in Table 3 . The values of the standard deviation between the corrupted and reconstructed parameters are obtained by replacing in Eq. (40) the values of exponent given in Table 2 applied to the design of the QUBIC instrument. It shows a very significant improvement on the level of the residual systematics after self-calibration, even for $1 \mathrm{~s}$.

\subsection{Finding limits}

Accordingly Eq. (34), in an ideal case, in the absence of systematic effects, the powers measured on the $x$ and $y$ polarized focal planes after demodulation of the half-wave plate are

$$
\begin{aligned}
\left(\begin{array}{c}
T_{\mathrm{O}}^{\eta} \\
T_{\mathrm{C}}^{\eta} \\
T_{\mathrm{S}}^{\eta}
\end{array}\right)= & \left(\begin{array}{c}
S_{I}^{\eta} \\
S_{Q}^{\eta} \\
S_{U}^{\eta}
\end{array}\right)=\int\left(\begin{array}{ccc}
B_{q, s}^{\eta}\left(\hat{n}_{\mathrm{p}}\right) & 0 & 0 \\
0 & \epsilon^{\eta} B_{q, s}^{\eta}\left(\hat{n}_{\mathrm{p}}\right) & 0 \\
0 & 0 & \epsilon^{\eta} B_{q, s}^{\eta}\left(\hat{n}_{\mathrm{p}}\right)
\end{array}\right) \\
& \bullet\left(\begin{array}{c}
I\left(\hat{n}_{\mathrm{p}}\right) \\
Q\left(\hat{n}_{\mathrm{p}}\right) \\
U\left(\hat{n}_{\mathrm{p}}\right)
\end{array}\right) \mathrm{d} v \mathrm{~d} \mathrm{~d} \hat{d}_{q} d \hat{n}_{\mathrm{p}}
\end{aligned}
$$

with $\epsilon^{x}=1$ for the polarization $x, \epsilon^{y}=-1$ for the polarization $y, T_{\mathrm{O}}, T_{\mathrm{C}}$, and $T_{\mathrm{S}}$ refer to a constant, cosine, and sine terms obtained after the demodulation of the half-wave plate, $S_{I}, S_{Q}$, and $S_{U}$ refer to the synthesized images for each Stokes parameter $I, Q$, and $U$ and $B_{q, \mathrm{~s}}^{\eta}\left(\hat{n}_{\mathrm{p}}\right)$ the synthesized beam. The integrations are performed over the bandwidth of the instrument, over the surface of the bolometer, and over the sky direction.

For a real instrument, in the case where the half-wave plate is located before the horns, leakages from $Q$ to $U$ and from $U$ to $Q$ appear. Using the self-calibration simulation, one can estimate the leakage from $Q$ into $U$ and from $U$ into $Q$ by calculating the standard deviation of the difference between the ideal and corrupted parameters $\sigma_{\text {id-corr }}^{I}, \sigma_{\text {id-corr }}^{Q}, \sigma_{\text {id-corr }}^{U}, \sigma_{\text {id-corr }}^{Q U}$, and $\sigma_{\text {id-corr }}^{U Q}$ 
A\&A 550, A59 (2013)

Table 2. Results of the self-calibration simulation for an instrument with 9 horns, 9 bolometers, and 10 pointings.

\begin{tabular}{lcccccccc}
\hline \hline & \multicolumn{3}{c}{$t_{\mathrm{b}}=1 \mathrm{~s}$} & \multicolumn{4}{c}{$t_{\mathrm{b}}=100 \mathrm{~s}$} \\
\hline Parameters & $\alpha$ & $\beta$ & $\gamma$ & $\Phi$ & $\alpha$ & $\beta$ & $\gamma$ & $\Phi$ \\
\hline$\alpha_{i q}^{\eta}$ & 0.74 & 0.99 & 0.75 & 0.84 & 0.97 & 1.55 & 1.22 & 1.06 \\
$\hat{n}_{\mathrm{p}}$ & 0.46 & 0.37 & 0.24 & 0.73 & 0.75 & 0.70 & 1.02 & 1.09 \\
$\vec{x}_{i}$ & 0.58 & 0.66 & 0.97 & 0.82 & 0.76 & 1.04 & 1.28 & 1.18 \\
$g_{\eta}\left(\vec{x}_{i}\right)$ & 0.91 & 1.06 & 0.83 & 0.48 & 1.04 & 1.52 & 0.45 & 0.75 \\
$e_{\eta}\left(\vec{x}_{i}\right)$ & 0.77 & 1.18 & 0.36 & 0.65 & 1.01 & 1.16 & 0.63 & 0.99 \\
$h_{\eta}$ & 0.55 & 0.67 & 0.12 & 0.59 & 0.78 & 0.85 & 0.25 & 0.78 \\
$\xi_{\eta}$ & 0.64 & 0.58 & 0.11 & 0.38 & 1.11 & 0.84 & 0.46 & 0.80 \\
\hline
\end{tabular}

Notes. The recovered parameters are given in the first column. Following the power law given by Eq. (40), one can calculate the exponent for each variable: the exponent $\alpha$ for the number of horns, the exponent $\beta$ for the number of bolometers, the exponent $\gamma$ for the number of pointings, and $\Phi$ the number of baselines per pointing. This work was done for two different measuring times per baseline $t_{\mathrm{b}}=1 \mathrm{~s}$ and $t_{\mathrm{b}}=100 \mathrm{~s}$ and with 40 realisations of the simulation.

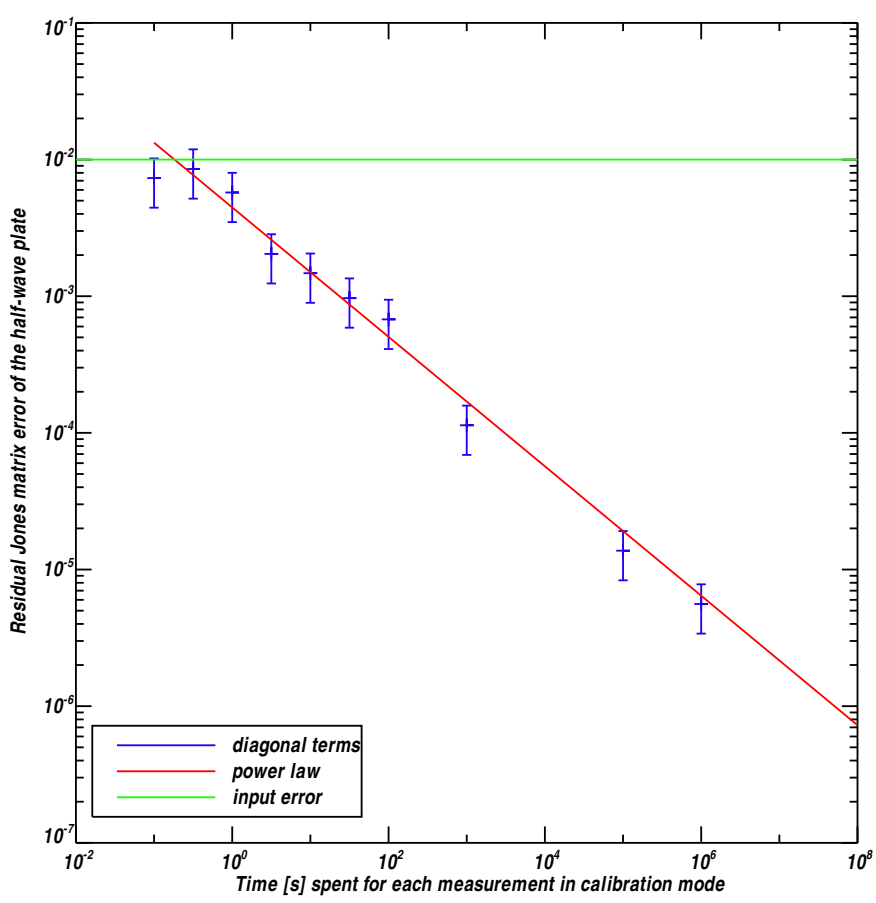

Fig. 4. Results of the self-calibration simulation for the diagonal terms of the Jones matrix of the half-wave plate. We show the residual error (in blue) on these parameters as a function of the measuring time spent on each baseline on the calibration mode. The red line represents a power law of the shape $\sim \frac{N E T}{\sqrt{t_{b}} \sqrt{2}}$ with $N E T$ the noise equivalent temperature of the bolometers, $T$ the temperature of the polarized source, and $t_{\mathrm{b}}$ the measuring time spent on each baseline. This law gives the limit of the relative accuracy that can be achieved on the systematic parameters according to the measuring time spent per baseline. The green line represents the input error on the systematic effects of the half-wave plate in the simulation given in Table 1.

(without self-calibration) and of the difference between the corrupted and recovered parameters $\sigma_{\text {corr-rec }}^{Q}, \sigma_{\text {corr-rec }}^{U}, \sigma_{\text {corr-rec }}^{Q U}$, and $\sigma_{\text {corr-rec }}^{U Q}$ (with self-calibration).

In the case without self-calibration, Eq. (41) becomes

$$
\left(\begin{array}{c}
\Delta T_{\mathrm{O}} \\
\Delta T_{\mathrm{C}} \\
\Delta T_{\mathrm{S}}
\end{array}\right)=\left(\begin{array}{ccc}
\sigma_{\text {id-corr }}^{I} & 0 & 0 \\
0 & \sigma_{\text {id-corr }}^{Q} & \sigma_{\text {id-corr }}^{U Q} \\
0 & \sigma_{\text {id-corr }}^{Q U} & \sigma_{\text {id-corr }}^{U}
\end{array}\right) \bullet\left(\begin{array}{c}
I\left(\hat{n}_{\mathrm{p}}\right) \\
Q\left(\hat{n}_{\mathrm{p}}\right) \\
U\left(\hat{n}_{\mathrm{p}}\right)
\end{array}\right) .
$$

In the case with self-calibration, Eq. (41) becomes

$$
\left(\begin{array}{c}
\Delta T_{\mathrm{O}} \\
\Delta T_{\mathrm{C}} \\
\Delta T_{\mathrm{S}}
\end{array}\right)=\left(\begin{array}{ccc}
\sigma_{\text {corr-rec }}^{I} & 0 & 0 \\
0 & \sigma_{\text {corr-rec }}^{Q} & \sigma_{\text {corr-rec }}^{U Q} \\
0 & \sigma_{\text {corr-rec }}^{Q U} & \sigma_{\text {corr-rec }}^{U}
\end{array}\right) \bullet\left(\begin{array}{c}
I\left(\hat{n}_{\mathrm{p}}\right) \\
Q\left(\hat{n}_{\mathrm{p}}\right) \\
U\left(\hat{n}_{\mathrm{p}}\right)
\end{array}\right) .
$$

With Eqs. (42) and (43), one can observe that instrument errors and systematic effects induce leakage from $Q$ to $U$ and from $U$ to $Q$ but also alter the polarization amplitude. It is assumed that there is no correlation of the errors in the synthesized beam $B_{q, \mathrm{~s}}^{\eta}\left(\hat{n}_{\mathrm{p}}\right)$ across the bolometers, and the simulation is realized for a point source so that the sky convolution can be ignored.

It is interesting to focus on the B-mode power spectrum in order to estimate the E-B mixing and to constrain the E-mode leakage in the B-mode power spectrum. In general, one can define the vector $\Delta \vec{S}$ that defines the errors on Stokes parameters

$\overrightarrow{\Delta S}=\left(\begin{array}{ccc}\Delta_{I I} & \Delta_{I Q} & \Delta_{I U} \\ \Delta_{Q I} & \Delta_{Q Q} & \Delta_{Q U} \\ \Delta_{U I} & \Delta_{U Q} & \Delta_{U U}\end{array}\right) \cdot \vec{S}$

where $\Delta \vec{S}=\vec{S}^{\text {rec }}-\vec{S}^{\text {corr }}$, and $\vec{S}$ corr is the vector of measured Stokes parameters and $\vec{S}$ rec is the vector of the Stokes parameters obtained with the self-calibration method. For the QUBIC instrument, there is no leakage of $I$ to $Q$ and $U$ because the halfwave plate is in front of the instrument so, Eq. (44) becomes

$\Delta \vec{S}=\left(\begin{array}{ccc}0 & 0 & 0 \\ 0 & \Delta_{Q Q} & \Delta_{Q U} \\ 0 & \Delta_{U Q} & \Delta_{U U}\end{array}\right) \bullet \vec{S}$

where $\Delta_{Q Q}, \Delta_{Q U}, \Delta_{U Q}$, and $\Delta_{U U}$ are the errors between the synthesized beam without the self-calibration method and the one after applying the self-calibration method. Following Appendix B and Eq. (45), one can write the error terms as $\Delta_{Q Q}=1+\epsilon, \Delta_{Q U}=\rho, \Delta_{U Q}=-\rho$, and $\Delta_{U U}=1+\epsilon$ where the complex term $\epsilon$ changes the amplitude of polarization and $\rho$ mixes both the $Q$ and $U$ Stokes parameters. One can define the error matrix on the $Q$ and $U$ Stokes parameters as $\mathbf{M}_{\mathbf{s}}-11$ where the matrix $\mathbf{M}_{\mathbf{s}}$ is given by

$\mathbf{M}_{\mathbf{s}}=\left(\begin{array}{cc}1+\epsilon & \rho \\ -\rho & 1+\epsilon\end{array}\right)$.

To go further, one can estimate the leakage from E to B-mode and give a constraint on the value of the tensor-to-scalar ratio $r$. 
Table 3. Results of the self-calibration simulation for the QUBIC instrument with 400 horns, $2 \times 1024$ bolometers array, 1000 pointings, and all baseline measurements.

\begin{tabular}{cccccc}
\hline \hline Parameters & $t_{\mathrm{b}}=0 \mathrm{~s}$ & \multicolumn{2}{c}{$t_{\mathrm{b}}=1 \mathrm{~s}$} & \multicolumn{2}{c}{$t_{\mathrm{b}}=100 \mathrm{~s}$} \\
\hline & $\sigma_{\text {id-corr }}$ & $\sigma_{\text {corr-rec }}$ & ratio & $\sigma_{\text {corr-rec }}$ & Ratio \\
\hline$\alpha_{i q}^{\eta}$ & 0.004 & $8.48 \times 10^{-5}$ & 47 & $1.87 \times 10^{-6}$ & 2140 \\
$\hat{n}_{\mathrm{p}}$ & 0.15 & $1.41 \times 10^{-3}$ & 106 & $3.26 \times 10^{-5}$ & 4596 \\
$\vec{x}_{i}$ & $100 . \times 10^{-6}$ & $5.86 \times 10^{-5}$ & 17 & $2.27 \times 10^{-8}$ & 4402 \\
$g_{\eta}\left(\vec{x}_{i}\right)$ & 0.0001 & $1.36 \times 10^{-6}$ & 73 & $1.22 \times 10^{-8}$ & 8182 \\
$e_{\eta}\left(\vec{x}_{i}\right)$ & 0.0001 & $1.09 \times 10^{-6}$ & 92 & $1.20 \times 10^{-8}$ & 8280 \\
$h_{\eta}$ & 0.01 & $1.18 \times 10^{-4}$ & 84 & $7.27 \times 10^{-6}$ & 1375 \\
$\xi_{\eta}$ & 0.01 & $1.24 \times 10^{-4}$ & 80 & $5.81 \times 10^{-6}$ & 1722 \\
\hline
\end{tabular}

Notes. The recovered parameters are given in the first column. In the second column, one can see the value of the standard deviation between the ideal and corrupted parameters (without self-calibration). The third and fifth columns give the value of the standard deviation between the corrupted and reconstructed parameters (with self-calibration). The benefit obtained after applying the self-calibration method is given in the fourth and sixth columns: this is the ratio between the value of the standard deviation of the third and the second columns for $t_{\mathrm{b}}=1 \mathrm{~s}$ and of the fifth and the second columns for $t_{\mathrm{b}}=100 \mathrm{~s}$.

An error in diagonal terms $\Delta_{Q Q}$ and $\Delta_{U U}$ will affect the amplitude of the $\mathrm{E}$ and $\mathrm{B}$-mode power spectrum. The nondiagonal terms $\Delta_{Q U}$ and $\Delta_{U Q}$ result in a leakage from the $\mathrm{E}$ to B-mode power spectrum (or the B to E-mode power spectrum). To have a constraint on the B-mode, as the E-mode is far above that of the B-mode in amplitude, one can use the equation derived by (Rosset et al. 2010) obtained with the first-order approximation

$\Delta C l^{\mathrm{BB}}=C l^{\mathrm{BB}, \text { meas }}-C l^{\mathrm{BB}}=2 \epsilon C l^{\mathrm{BB}}+\rho^{2} C l^{\mathrm{EE}}$

where $\Delta C l^{\mathrm{BB}}$ is the error on the $\mathrm{B}$-mode power spectrum $C l^{\mathrm{BB}}$ and $C l^{\mathrm{EE}}$ is the E-mode power spectrum, and we suppose $\epsilon$ is small. One can refer to Appendix B for the explicit details of this equation. It shows that the uncertainty on parameter $\rho$ must be lower than $0.5 \%$ to have a leakage from $\mathrm{E}$ to B-mode lower than $10 \%$ of the expected B-mode power spectrum $C l^{\mathrm{BB}}$ for a tensor-to-scalar ratio of $r=0.01$ for $l<100$.

From Eq. (47), one can estimate the leakage from the E-mode into the B-mode power spectrum given by the term $\rho^{2} C l^{\mathrm{EE}}$. Figure 5 represents the error on the B-mode power spectrum $\Delta C_{l}$ as a function of the multipoles. The leakage from the E-mode into the B-mode is therefore significantly reduced by applying the self-calibration procedure, even with a modest $1 \mathrm{~s}$ per baseline (corresponding to a full day dedicated to selfcalibration). The leakage can be further reduced by spending more time on self-calibration.

\section{Conclusion}

Bolometric interferometry differs considerably from standard radio interferometry in the sense that its primary goal is not to reach a good angular resolution but to achieve high statistical sensitivity and good control of systematic effects. In this perspective, redundancy turns out to be the crucial property to fulfill these two objectives, as shown in Charlassier et al. (2009) and in the present article.

In this paper, we have shown that with a polarized calibration source and the use of the successive observation of this source with all pairs of horns of the interferometer (self-calibration), one can have low and controllable instrumental systematic effects. Redundant baselines should give the same signal if they are free of systematics. By modeling the instrument systematics with a set of parameters (Jones matrices, location of the horns, beams), one can use the measurements of the different baselines to solve a nonlinear system that allows the systematic effects

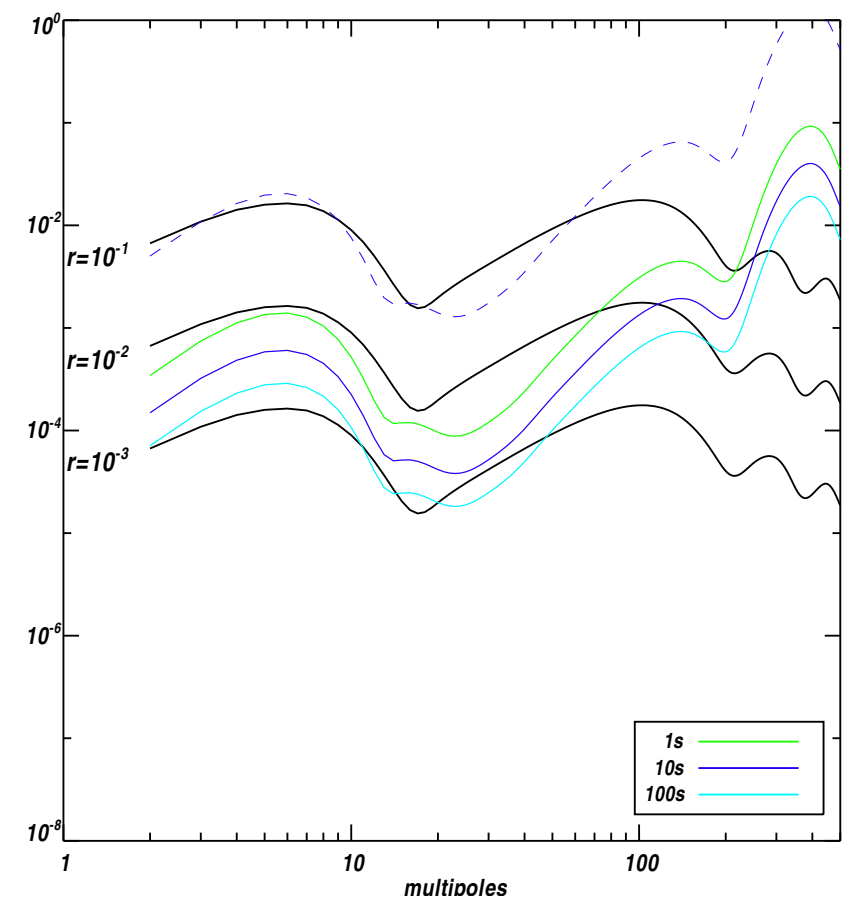

Fig. 5. $\Delta C_{l}$ due to leakage from E-mode for different times on measurements per baseline $t_{\mathrm{b}}=1 \mathrm{~s}, 10 \mathrm{~s}$ and, $100 \mathrm{~s}$ for the QUBIC instrument. The colored solid lines represent the leakage after applying the self-calibration method for different measuring times per baseline. The dashed line represents the error on the B-mode power spectrum without self-calibration method. The black lines are the primordial B-mode spectrums for $r=10^{-1}, r=10^{-2}$, and $r=10^{-3}$.

parameters to be determined with an accuracy that, besides the correctness of the modelling, is only limited by the photon noise, hence by the time spent on self-calibration. The more horns and bolometers in the array, the more efficient the self-calibration procedure.

The resolution of the system is CPU-intensive for large bolometric interferometers and should be implemented on massively parallel computers in the future. Using simulations with various horns and bolometer arrays of moderate sizes, we have obtained a scaling law that allows us to extrapolate the accuracy of self-calibration to the QUBIC instrument with 400 horns, $2 \times 1024$ bolometer arrays and 1000 pointing directions towards 
the calibration source. We find that with a few seconds per baseline (corresponding to a few days spent on self-calibration), knowledge of the instrumental systematic effects parameters can be improved by at least two orders of magnitude, allowing minimization of the leakage from $\mathrm{E}$ into $\mathrm{B}$ polarization down to a tolerable level. This can be improved by spending more time on self-calibration.

The idea of developing bolometric interferometry was motivated by bringing together the imager exquisite sensitivity allowed by bolometer arrays and the ability to handle instrumental systematic effects allowed by interferometers. Bolometric interferometers have been shown to have a sensitivity similar to that of imagers (Hamilton 2008; QUBIC collaboration 2010), while we have shown in the present article that the self calibration allows achieving an excellent handling of systematic effects that has no equivalent with an imager.

Acknowledgements. The authors are grateful to the QUBIC collaboration and Craig Markwardt for MPFIT. They would like to thank Gael Roudier for his help and the referee for an attentive reading. This work was supported by Agence Nationale de la Recherche (ANR), Centre National de la Recherche Scientifique (CNRS), and la région d'Ile de France.

\section{Appendix A: Measuring the bolometer power for two opened horns is equivalent to measuring the bolometer power when all horns are open except the horn $i$ and $j$}

The power collected by the bolometer $q$ for the opened horns $i$ and $j$ without polarization is

$S_{i j}=C_{i}+C_{j}+2 \operatorname{Re}\left(\alpha_{i q} \alpha_{j q}^{*} \beta_{i} \beta_{j}^{*}\right)$,

which can be written as

$S_{i j}=\left|p_{i}\right|^{2}+\left|p_{j}\right|^{2}+2 \operatorname{Re}\left(p_{i} p_{j}^{*}\right)$.

The total power measured for all the baselines can be expressed as

$S_{\text {tot }}=\left|\sum_{m} p_{m}\right|^{2}=\sum_{m}\left|p_{m}\right|^{2}+\sum_{m \neq l} 2 \operatorname{Re}\left(p_{m} p_{l}^{*}\right)$.

The power measured by a bolometer $q$ for all horns opened except the horn $i$ is

$C_{-i}=\left|\sum_{j \neq i} p_{j}\right|^{2}=S_{\mathrm{tot}}-C_{i}-\sum_{k \neq i} 2 \operatorname{Re}\left(p_{i} p_{k}^{*}\right)$.

The power measured by a bolometer $q$ for all horns opened except the horn $j$ is

$C_{-j}=\left|\sum_{i \neq j} p_{j}\right|^{2}=S_{\text {tot }}-C_{j}-\sum_{k \neq j} 2 \operatorname{Re}\left(p_{j} p_{k}^{*}\right)$.

The power measured by a bolometer $\mathrm{q}$ for all baselines opened, except the baseline formed by the horns $i$ and $j$, is

$S_{-i j}=S_{\text {tot }}-C_{i}-C_{j}-\sum_{k \neq i} 2 \operatorname{Re}\left(p_{i} p_{k}^{*}\right)-\sum_{k \neq j} 2 \operatorname{Re}\left(p_{j} p_{k}^{*}\right)+2 \operatorname{Re}\left(p_{i} p_{j}^{*}\right)$

with

$$
\begin{aligned}
2 \operatorname{Re}\left(p_{i} p_{j}^{*}\right)=S_{\text {tot }}+S_{-i j}-S_{\text {tot }}+C_{i}+\sum_{k \neq i} 2 \operatorname{Re}\left(p_{i} p_{k}^{*}\right)-S_{\text {tot }} \\
+C_{j}+\sum_{k \neq j} 2 \operatorname{Re}\left(p_{j} p_{k}^{*}\right) .
\end{aligned}
$$

Finally, one can find

$2 \operatorname{Re}\left(p_{i} p_{j}^{*}\right)=S_{\text {tot }}+S_{-i j}-S_{\text {tot }}-C_{-i}-C_{-j}$.

So:

$S_{-i j}=C_{-i}+C_{-j}+2 \operatorname{Re}\left(p_{i} p_{j}^{*}\right)$.

\section{Appendix B: Error on E and B-mode power spectra}

One can define the Stokes parameters in spin-2 spherical harmonics base

$Q(\hat{n}) \pm \mathrm{i} U(\hat{n})=\sum_{l m} a_{ \pm 2 l m \pm 2} Y_{l m}(\hat{n})$

where $Q$ and $U$ are defined at each direction $\hat{n}$.

It is convenient to introduce the linear combinations

$a_{l m}^{E}=-\frac{a_{2 l m}+a_{-2 l m}}{2}$

$a_{l m}^{B}=\mathrm{i} \frac{a_{2 l m}-a_{-2 l m}}{2}$.

One can define the two scalar fields

$E(\hat{n})=\sum_{l=0}^{\infty} \sum_{m=-l}^{l} a_{l m}^{\mathrm{E}} Y_{l m}(\hat{n})$

$B(\hat{n})=\sum_{l=0}^{\infty} \sum_{m=-l}^{l} a_{l m}^{\mathrm{B}} Y_{l m}(\hat{n})$

Using the coefficients $a_{l m}^{\mathrm{E}}$ and $a_{l m}^{\mathrm{B}}$, one can construct the angular power spectrum $C_{l}^{\mathrm{EE}}$ and $C_{l}^{\mathrm{BB}}$ as

$C_{l}^{\mathrm{EE}}=\left\langle\left|a_{\mathrm{lm}}^{\mathrm{E}}\right|^{2}\right\rangle$

$C_{l}^{\mathrm{BB}}=\left\langle\left|a_{\mathrm{lm}}^{\mathrm{B}}\right|^{2}\right\rangle$.

One can express the $Q$ and $U$ Stokes parameters as a function of the coefficients $a_{l m}^{\mathrm{E}}$ and $a_{l m}^{\mathrm{B}}$

$Q(\hat{n})=\frac{1}{2} \sum_{l, m}\left[\left(\mathrm{i} a_{l m}^{\mathrm{B}}-a_{l m}^{\mathrm{E}}\right)_{-2} Y_{l m}(\hat{n})+\left(-\mathrm{i} a_{l m}^{\mathrm{B}}-a_{l m}^{\mathrm{E}}\right)_{+2} Y_{l m}(\hat{n})\right]$

$U(\hat{n})=\frac{1}{2} \sum_{l, m}\left[\mathrm{i}\left(\mathrm{i} a_{l m}^{\mathrm{B}}-a_{l m}^{\mathrm{E}}\right)_{-2} Y_{l m}(\hat{n})+\left(-\mathrm{i} a_{l m}^{\mathrm{B}}-a_{l m}^{\mathrm{E}}\right)_{+2} Y_{l m}(\hat{n})\right]$.

Using Eqs. (B.4), (B.5), (B.8), and (B.9), one can express the coefficients $a_{l m}^{E}$ and $a_{l m}^{B}$ as a function of the Stokes parameters $Q$ and $U$

$\left(\begin{array}{l}a_{l m}^{\mathrm{E}} \\ a_{l m}^{\mathrm{B}}\end{array}\right)=\int \mathbf{M}_{\mathbf{a l m}}\left(\begin{array}{l}Q(\hat{n}) \\ U(\hat{n})\end{array}\right) \mathrm{d} \Omega$

where the integration is taken over the whole sky, and

$\mathbf{M}_{\mathbf{a l m}}=\left(\begin{array}{ccc}-\left({ }_{+2} Y_{l m}(\hat{n})+_{-2}\right. & \left.Y_{l m}(\hat{n})\right) & -\mathrm{i}\left({ }_{+2} Y_{l m}(\hat{n})-{ }_{-2} Y_{l m}(\hat{n})\right) \\ \mathrm{i}\left({ }_{+2} Y_{l m}(\hat{n})--_{-2}\right. & Y_{l m}(\hat{n}) & -\left({ }_{+2} Y_{l m}(\hat{n})+{ }_{-2} Y_{l m}(\hat{n})\right)\end{array}\right)$.

In the case of a bolometric interferometer, globar errors on synthesized beam will affect the amplitude of polarization and mix the $Q$ and $U$ Stokes parameters. 
To model systematic errors, one can introduce a Jones matrix that describes the propagation of radiation through a receiver

$\overrightarrow{E_{r}}=\overrightarrow{\mathbf{J} E}=\left(\begin{array}{cc}1-g_{x} & e_{x} \\ e_{y} & 1-g_{y}\end{array}\right) \vec{E}$

where the gain $g_{\eta}$ and the leakage $e_{\eta}$ are complex values.

From this relation, one can construct the Mueller matrix $\mathbf{M}$, which tells us how the Stokes vector $\vec{S}$ transforms

$\vec{S}_{r}=\mathbf{A}\left(\mathbf{J} \otimes \mathbf{J}^{*}\right) \mathbf{A}^{-1} \vec{S}=\mathbf{M} \vec{S}$

where $\mathbf{A}=\left[\begin{array}{cccc}1 & 0 & 0 & 1 \\ 1 & 0 & 0 & -1 \\ 0 & 1 & 1 & 0 \\ 0 & i & -i & 0\end{array}\right]$

$C l^{\mathrm{EE}, \text { meas }}=C l^{\mathrm{EE}}+\epsilon(2+\epsilon) C l^{\mathrm{EE}}+\rho^{2} C l^{\mathrm{BB}} \simeq C l^{\mathrm{EE}}+2 \epsilon C l^{\mathrm{EE}}+\rho^{2} C l^{\mathrm{BB}}$

and $\vec{S}_{r}$ is the outgoing Stokes vector and $\mathbf{M}=\mathbf{A}\left(\mathbf{J} \otimes \mathbf{J}^{*}\right) \mathbf{A}^{-1}$ is the resulting error matrix.

We are only interested in the $Q$ and $U$ Stokes parameters. In this case, the error matrix $\mathbf{M}$ becomes

$\mathbf{M}=\left(\begin{array}{ll}M_{Q Q} & M_{Q U} \\ M_{U Q} & M_{U U}\end{array}\right)$.

The first order of this matrix $\mathbf{M}$ is

$M_{Q Q}=1+g_{x}+g_{y}+g_{x}^{*}+g_{y}^{*}$

$M_{U Q}=e_{y}+e_{y}^{*}-e_{x}-e_{x}^{*}$

$M_{Q U}=e_{x}+e_{x}^{*}-e_{y}-e_{y}^{*}$

$M_{U U}=1+g_{x}+g_{y}+g_{x}^{*}+g_{y}^{*}$.

One can generalize this error matrix for Jones matrix of any component and rewrite it as $\mathbf{M}_{\mathbf{s}}-\mathbb{1 1}$ where

$\mathbf{M}_{\mathbf{s}}=\left(\begin{array}{cc}1+\epsilon & \rho \\ -\rho & 1+\epsilon\end{array}\right)$

where the complex term $\epsilon$ describes the error of the amplitude of polarization and the complex term $\rho$ mixes the $Q$ and $U$ Stokes parameters.

In this case, Eq. (B.10) becomes

$\left(\begin{array}{l}a_{l m}^{\mathrm{E}, \text { meas }} \\ a_{l m}^{\mathrm{B}, \text { meas }}\end{array}\right)=\mathbf{M}_{\mathrm{alm}} \mathbf{M}_{\mathbf{s}}\left(\begin{array}{l}Q(\hat{n}) \\ U(\hat{n})\end{array}\right)$

where the coefficients $a_{1 \mathrm{~m}}^{\mathrm{E}, \text { meas }}$ and $a_{l m}^{\mathrm{B} \text {,meas }}$ include systematic effects.

In terms of power spectra, an error in polarization amplitude will affect the amplitude of the E and B-mode power spectra,
$C l^{\mathrm{BB}, \text { meas }}=C l^{\mathrm{BB}}+\epsilon(2+\epsilon) C l^{\mathrm{BB}}+\rho^{2} C l^{\mathrm{EE}} \simeq C l^{\mathrm{BB}}+2 \epsilon C l^{\mathrm{BB}}+\rho^{2} C l^{\mathrm{EE}}$,

and an error that mixes the $Q$ and $U$ Stokes parameters leads to a leakage from the $\mathrm{E}$ to B-mode (and from the B to E-mode).

One can easily find that with systematic effects, the coefficients $a_{l m}^{\mathrm{E}}$ and $a_{l m}^{\mathrm{B}}$ can be expressed as

$a_{l m}^{\mathrm{E}, \text { meas }}=a_{l m}^{\mathrm{E}}+\epsilon a_{l m}^{\mathrm{E}}-\rho a_{l m}^{\mathrm{B}}$

$a_{l m}^{\mathrm{B} \text {,meas }}=a_{l m}^{\mathrm{B}}+\epsilon a_{l m}^{\mathrm{B}}+\rho a_{l m}^{\mathrm{E}}$

where $\epsilon$ is the error in amplitude and $\rho$ the error of polarization leakage. Using Eqs. (B.6) and (B.7), one can obtain

where we suppose $\epsilon$ is small, $C l^{\mathrm{EE} \text {,meas }}$ and $C l^{\mathrm{BB} \text {,meas }}$ are the $\mathrm{E}$ and B-mode power spectra including systematic effects, $C l^{\mathrm{EE}}$ and $C l^{\mathrm{BB}}$ the input power spectra, the complex term $\epsilon$ describes the error of amplitude of the B-mode power spectrum, and the complex term $\rho$ results in a leakage from the $\mathrm{E}$ to $\mathrm{B}$-mode power spectrum.

To focus on the B-mode, one can define the error on $C l^{\mathrm{BB}}$ power spectrum

$\Delta C l^{\mathrm{BB}}=C l^{\mathrm{BB}, \text { meas }}-C l^{\mathrm{BB}}=2 \epsilon C l^{\mathrm{BB}}+\rho^{2} C l^{\mathrm{EE}}$.

\section{References}

Bock, J., Church, S., Devlin, M., et al. 2006 [arXiv: astro-ph/0604101] Bunn, E. F. 2007, Phys. Rev. D, 75, 083517

Charlassier R. 2010, Ph.D., University Paris-Diderot

Charlassier, R., Hamilton, J., Bréelle, E., et al. 2009, A\&A, 497, 963

Charlassier, R., Bunn, E. F., Hamilton, J., Kaplan, J., \& Malu, S. 2010, A\&A, 514, A37

Hamilton, J. -C., Charlassier , R., Cressiot , C., et al. 2008, A\&A, 491, 923

Hu, W., Hedman, M. M., \& Zaldarriaga, M. 2003, Phys. Rev. D, 67, 043004

Hyland, P., Follin, B., \& Bunn, E. F. 2009, MNRAS, 393, 53

Kovac, J., Leitch, E. M., Pryke, C., et al. 2002, Nature, 420, 772

Liu, A., Tegmark, M., Morrison, S., Lutomirski, A., \& Zaldarriaga, M. 2010, MNRAS, 408, 1029

Markwardt, C. B. 2009, in ASP Conf. Ser. 411, eds. D. A. Bohlender, D. Durand, \& P. Dowler, 251

Noordam, J. E., \& de Bruyn, A. G. 1982, Nature, 299, 597

O'Dea, D., Challinor, A., \& Johnson, B. R. 2007, MNRAS, 376, 1767

Pearson, T. J., \& Readhead, A. C. S. 1984, ARA\&A, 22, 97

Readhead, A. C. S., Myers, S. T., Pearson, T. J., et al. 2004, Science, 306, 836

Rosset, C., Tristram, N., Ponthieu, N., et al. 2010, A\&A, 520, A13

Tegmark, M., \& Zaldarriaga, M. 2009, Phys. Rev. D, 79, 083530

Tegmark, M., \& Zaldarriaga, M. 2012, Phys. Rev. D, 82, 103501

The QUBIC Collaboration 2010, Astropart. Phys., 34

Timbie, P. T., Tucker, G. S., Ade, P. A. R., et al. 2006, New Astron. Rev., 50, 999

Tucker, G. S., Kim, J., Timbie, P., et al 2003, New Astron. Rev., 47, 1173

Wieringa, M. 1991, in IAU Colloq. 131: Radio Interferometry. Theory, Techniques, and Applications, eds. T. J. Cornwell, \& R. A. Perley, ASP Conf. Ser., 19, 192196 